\title{
Dynamic Fluorescence Microscopy of Cellular Uptake of Intercalating Model Drugs by Ultrasound-Activated Microbubbles
}

\author{
B.H.A. Lammertink, ${ }^{1}$ R. Deckers, ${ }^{1}$ M. Derieppe, ${ }^{1}$ I. De Cock, ${ }^{2}$ I. Lentacker, ${ }^{2}$ G. Storm, ${ }^{3,4}$ \\ C.T.W. Moonen, ${ }^{1}$ C. Bos ${ }^{1}$ \\ ${ }^{1}$ Imaging Division, UMC Utrecht, Heidelberglaan 100, 3584 CX, Utrecht, The Netherlands \\ ${ }^{2}$ Department of Pharmaceutics, Ghent University, Ghent, Belgium \\ ${ }^{3}$ Pharmaceutics Department, Utrecht University, Utrecht, The Netherlands \\ ${ }^{4}$ Targeted Therapeutics, MIRA Institute, University of Twente, Enschede, The Netherlands
}

\begin{abstract}
Purpose: The combination of ultrasound and microbubbles can facilitate cellular uptake of (model) drugs via transient permeabilization of the cell membrane. By using fluorescent molecules, this process can be studied conveniently with confocal fluorescence microscopy. This study aimed to investigate the relation between cellular uptake and fluorescence intensity increase of intercalating model drugs.

Procedures: SYTOX Green, an intercalating fluorescent dye that displays $>500$-fold fluorescence enhancement upon binding to nucleic acids, was used as a model drug for ultrasoundinduced cellular uptake. SYTOX Green uptake was monitored in high spatiotemporal resolution to qualitatively assess the relation between uptake and fluorescence intensity in individual cells. In addition, the kinetics of fluorescence enhancement were studied as a function of experimental parameters, in particular, laser duty cycle (DC), SYTOX Green concentration and cell line. Results: Ultrasound-induced intracellular SYTOX Green uptake resulted in local fluorescence enhancement, spreading throughout the cell and ultimately accumulating in the nucleus during the 9-min acquisition. The temporal evolution of SYTOX Green fluorescence was substantially influenced by laser duty cycle: continuous laser (100 \% DC) induced a 6.4-fold higher photobleaching compared to pulsed laser (3.3\% DC), thus overestimating the fluorescence kinetics. A positive correlation of fluorescence kinetics and SYTOX Green concentration was found, increasing from $0.6 \times 10^{-3}$ to $2.2 \times 10^{-3} \mathrm{~s}^{-1}$ for 1 and $20 \mu \mathrm{M}$, respectively. Finally, C6 cells displayed a 2.4-fold higher fluorescence rate constant than FaDu cells.

Conclusions: These data show that the temporal behavior of intracellular SYTOX Green fluorescence enhancement depends substantially on nuclear accumulation and not just on cellular uptake. In addition, it is strongly influenced by the experimental conditions, such as the laser duty cycle, SYTOX Green concentration, and cell line.
\end{abstract}

Key Words: Model drug, Fluorescence, Confocal microscopy, Ultrasound, Microbubbles, Drug delivery

Electronic supplementary material The online version of this article (doi:10.1007/s11307-016-1042-x) contains supplementary material, which is available to authorized users.

Correspondence to: C. Bos; e-mail: c.bos@umcutrecht.nl 


\section{Introduction}

The combination of ultrasound (US) and microbubbles (MB) is a non-invasive technique to enhance local drug concentration in vivo [1], without encapsulating the drug [2] or altering its physicochemical properties [3]. Ultrasound and microbubble (USMB)-induced cavitation has been demonstrated to transiently disturb the integrity of plasma membranes, increasing its permeability and thereby enhancing intracellular (model) drug uptake [4-6].

Several mechanisms have been proposed to explain this phenomenon, including pore formation $[7,8]$ and upregulation of endocytosis $[9,10]$. It has been demonstrated that the size of the (model) drug [9] and the acoustic pressure used in the experiment [10] affect the mode of cellular uptake.

Uptake of fluorescent model drugs can be assessed on a single-cell level using confocal fluorescence microscopy, allowing real-time monitoring of the intracellular spatial distribution of the model drug, in contrast to analytical techniques such as high-performance liquid chromatography. By analyzing single cells, confocal fluorescence microscopy can detect the heterogeneity of the underlying microbubble-cell interactions, as opposed to the cell population-based analytical techniques.

Intercalating fluorescent model drugs, such as SYTOX Green or propidium iodide (PI), are extensively used to study USMB-induced intracellular drug delivery [11, 12]. They are hydrophilic molecules that are normally not taken up by cells and become strongly fluorescent upon binding nucleic acids after entering the intracellular domain [16]. These properties make these compounds convenient for studying USMB-induced membrane permeabilization and drug uptake using conventional research techniques, e.g., fluorescence microscopy and flow cytometry [13-15].

Dedicated setups are increasingly being used to dynamically monitor USMB-induced cellular internalization of these model drugs in real-time by confocal fluorescence microscopy, aiming to give a quantitative description of the uptake kinetics [16-19]. However, when monitoring the kinetics of cellular internalization, results should be interpreted with caution, as the fluorescence intensity of these intercalating model drugs not only depends on cellular uptake but also on nucleic acid binding. In other words, the fluorescence intensity is no longer strictly proportional to the intracellular concentration, which may result in misinterpretation of the observed data.

Therefore, the research in this manuscript aimed to increase our understanding of the kinetics of fluorescence enhancement following USMB-induced cellular internalization of intercalating model drugs. This allows to improve the experimental design of future experiments as well as to guide the interpretation of observed results.

To this end, the study characterized the fluorescence intensity enhancement upon USMB-induced intracellular SYTOX Green uptake in vitro, as a function of the experimental parameters. USMB-induced SYTOX Green uptake was monitored in high spatiotemporal resolution in single cells using confocal fluorescence microscopy. In addition, the fluorescence kinetics were studied in a population of cells to assess the effect of laser duty cycle, SYTOX Green concentration, and cell line.

\section{Materials and Methods}

\section{Cell Culture}

Human melanoma (BLM) cells [20] were cultured in Dulbecco's modified Eagle's medium (DMEM) with Nutrient Mixture F12 (Gibco, Merelbeke, Belgium), supplemented with $10 \%$ fetal bovine serum (FBS) (Hyclone, Thermo Scientific, MA, USA), $20 \mathrm{U} / \mathrm{ml}$ penicillinstreptomycin (Gibco), $2 \mathrm{mM}$ L-glutamine (Gibco), and $10 \mathrm{mM}$ HEPES (Sigma-Aldrich ${ }^{\circledR}$ ). Human pharynx squamous cell carcinoma $(\mathrm{FaDu})$ cells (ATCC ${ }^{\circledR}$ HTB-43 ${ }^{\mathrm{TM}}$, LGC Standards GmbH, Wesel, Germany) were cultured in high-glucose DMEM (Sigma-Aldrich ${ }^{\circledR}$, St. Louis, MO, USA), supplemented with $10 \%(v / v)$ FBS (Sigma-Aldrich ${ }^{\circledR}$ ) and $1 \%$ non-essential amino acids (Sigma-Aldrich ${ }^{\circledR}$ ). Rat glioma (C6) cells (ATCC ${ }^{\circledR}$ CCL-107TM) were maintained in low-glucose DMEM (Sigma-Aldrich ${ }^{\circledR}$ ) supplemented with $10 \%$ FBS. Cells were cultured in standard cell culture flasks in a humidified incubator at $5 \% \mathrm{CO}_{2}$ and $37{ }^{\circ} \mathrm{C}$.

Ultrasound experiments with BLM cells were performed in OptiCells ${ }^{\mathrm{TM}}$ (Nunc, Thermo Scientific, MA, USA), wherein $1.3 \times 10^{6}$ cells were plated 1 day prior to the experiment. For ultrasound experiments with $\mathrm{FaDu}$ or $\mathrm{C} 6$ cells, $1 \times 10^{6}$ cells were seeded into CLINIcell ${ }^{\circledR}$ cell culture chambers (Mabio, Tourcoing, France) 2 days prior to the experiment, to ensure a confluent cell monolayer during the experiment. CLINIcells ${ }^{\circledR}$ were coated with Poly-L-Lysine (Sigma-Aldrich ${ }^{\circledR}$ ) before cell seeding for proper cell attachment.

\section{Microbubbles}

For the experiments with BLM cells, microbubbles composed of a DPPC and DSPE-PEG shell-encapsulating $\mathrm{C}_{4} \mathrm{~F}_{10}$ gas were prepared as described previously [10]. They were freshly prepared on the experimental day and kept for a maximum of $4 \mathrm{~h}$. In this setup, $40 \mu \mathrm{l}$ of microbubbles was added to a $10-\mathrm{ml}$ medium just before injection into the OptiCell $^{\mathrm{TM}}$. In the US experiments with FaDu and C6 cells, the ultrasound contrast agent SonoVue ${ }^{\mathrm{TM}}$ (Bracco, Milan, Italy) was used, as described previously [12].

\section{Chemicals}

SYTOX Green (Life Technologies ${ }^{\mathrm{TM}}$ Europe BV, Bleiswijk, Netherlands; excitation $(E x) /$ emission $(E m)=504 / 523 \mathrm{~nm})$ was used as a model drug. SYTOX Green shows little native fluorescence, but upon binding to nucleic acids, the fluorescence intensity enhances $>500$-fold. PI, another 
commonly used fluorescent model drug, was used to compare the fluorescence kinetics between the two model drugs. PI (Thermo Fisher Scientific) fluorescence intensity enhances 30-fold upon binding to nucleic acids. CellMask ${ }^{\mathrm{TM}}$ Deep Red plasma membrane stain (Thermo Fisher Scientific, Waltham, MA, USA; Ex/Em $=649 / 666 \mathrm{~nm}$ ) was used to visualize the plasma membrane in the swept field confocal microscopy experiments. In the same experiments, Hoechst 33342 (Sigma-Aldrich $\mathbb{R}$; Ex/Em $=350 / 461 \mathrm{~nm}$ ) was used to stain cell nuclei, which allowed for segmentation of the nucleus in the data analysis.

\section{Ultrasound Equipment}

Two ultrasound setups were used in these studies, each dedicated to its own confocal microscopy system. First, an ultrasound setup mounted on a swept field confocal microscope for real-time confocal recordings was used as described previously (Fig. 1, left panel) [10]. In this setup, BLM cells were sonicated for $5 \mathrm{~s}$ with $1.0 \mathrm{MHz}$ ultrasound at $15 \%$ duty cycle (DC), $1 \mathrm{kHz}$ pulse repetition frequency (PRF), and $100 \mathrm{kPa}$ peak negative pressure (PNP), as was calibrated with a needle hydrophone. These settings were based on previous work with this cell line [10].

A second ultrasound setup was used in combination with a fibered confocal fluorescence microscope, as reported before [16]. Here, $\mathrm{C} 6$ and $\mathrm{FaDu}$ cells were exposed to $1.5 \mathrm{MHz}$ ultrasound for $5 \mathrm{~s}$, at $10 \% \mathrm{DC}$ and $1 \mathrm{kHz}$ PRF. These settings were based on previous experience with $\mathrm{C} 6$ cells [16]. For SYTOX Green uptake experiments, FaDu or C6 cells were exposed to 350,600 and $850 \mathrm{kPa} \mathrm{PNP}$, as was calibrated by a fiber optic hydrophone (Fibre-optic Hydrophone System, Precision Acoustics).

\section{High-Resolution Monitoring of Ultrasound- Induced SYTOX Green Uptake in Single Cells}

Ultrasound-induced SYTOX Green uptake in BLM cells was monitored in detail using a swept field confocal microscope [10]. Cellular plasma membranes and nuclei were stained with $4 \mu \mathrm{g} / \mathrm{ml}$ CellMask $^{\mathrm{TM}}$ Deep Red and $20 \mu \mathrm{M}$ Hoechst, respectively, for $20 \mathrm{~min}$ prior to the uptake experiment. Then, $10 \mathrm{ml}$ Opti-MEM ${ }^{\circledR}$ (Gibco) with $50 \mu \mathrm{l}$ microbubbles and $2 \mu \mathrm{M}$ SYTOX Green was added to the OptiCell ${ }^{\mathrm{TM}}$, which was placed in the water bath, such that the bubbles to ascended towards the cells (Fig. 1). Recordings started $10 \mathrm{~s}$ prior to US exposure, and images were acquired for $9 \mathrm{~min}$. During post-processing of the data, the Hoechst signal was used to segment the nuclei, while a second region of interest was drawn manually in the cytosol. Subsequently, SYTOX Green fluorescence enhancement in the nucleus and the cytosol were analyzed in the Icy software [21]. Nuclear SYTOX Green signal was averaged every $20 \mathrm{~s}$ to minimize the effect of focal plane drift that occurred during acquisition.

\section{Monitoring of USMB-Induced SYTOX Green Uptake in a Population of Cells}

SYTOX Green uptake following USMB treatment was monitored in a population of cells using a fibered confocal fluorescence microscope (FCFM) (Cellvizio®, Mauna Kea Technologies, Paris, France), as previously described [22]. The FCFM microprobe has a field of view of $593 \times 593 \mu \mathrm{m}$, and a $488 \mathrm{~nm}$ laser was used to excite SYTOX Green at $0.5 \mathrm{~mW}$ laser power. As SYTOX Green fluorescence signal enhancement continued for 10-30 min, snapshots were recorded every $30 \mathrm{~s}$ for $30 \mathrm{~min}$.

\section{Influence of Laser-Induced Photobleaching on Fluorescence Kinetics}

First, the effect of photobleaching on the measured fluorescence intensity following USMB-induced SYTOX Green uptake was studied in FaDu cells. Cells were exposed to ultrasound in the presence of $2 \mu \mathrm{M}$ SYTOX Green and SonoVue ${ }^{\mathrm{TM}}$ microbubbles. SYTOX Green uptake was recorded using the FCFM, while the laser output was switched off between acquisitions $(3.3 \%$ duty cycle, henceforth termed pulsed laser) or with a laser that was continuously on (100\% duty cycle).

In order to separate photobleaching and uptake, confluent FaDu cells were chemically permeabilized with $0.5 \%$ Triton X100 for 1 min and then washed and fixed in $4 \%$ PFA during $15 \mathrm{~min}$ on ice. Subsequently, cells were incubated with $2 \mu \mathrm{M}$ SYTOX Green for $30 \mathrm{~min}$. Next, the staining solution was removed and fresh SYTOX Green-free PBS was added. The fluorescence signal of the SYTOX Green positive cells was recorded using the FCFM with pulsed and continuous laser.

\section{Concentration-Dependent Fluorescence Kinetics}

To assess the influence of dye concentration on the fluorescence kinetics, $\mathrm{FaDu}$ cells were exposed to ultrasound in the presence of $1,2,5$, or $10 \mu \mathrm{M}$ SYTOX Green and SonoVue ${ }^{\mathrm{TM}}$ microbubbles. To compare the fluorescence kinetics of SYTOX Green with PI, similar experiments were performed with $30 \mu \mathrm{M}$ PI, which was the minimum concentration required to obtain sufficient signal for kinetics quantification. Signal intensities of SYTOX Green or PI positive cells were recorded with pulsed laser on the FCFM. The relation between the fluorescence rate constant and SYTOX Green concentration was tested by linear regression.

\section{SYTOX Green Uptake in Chemically Permeabilized Cells}

The fluorescence kinetics of SYTOX Green following internalization in chemically permeabilized cells was measured by spectrofluorometry. To reach a confluent 


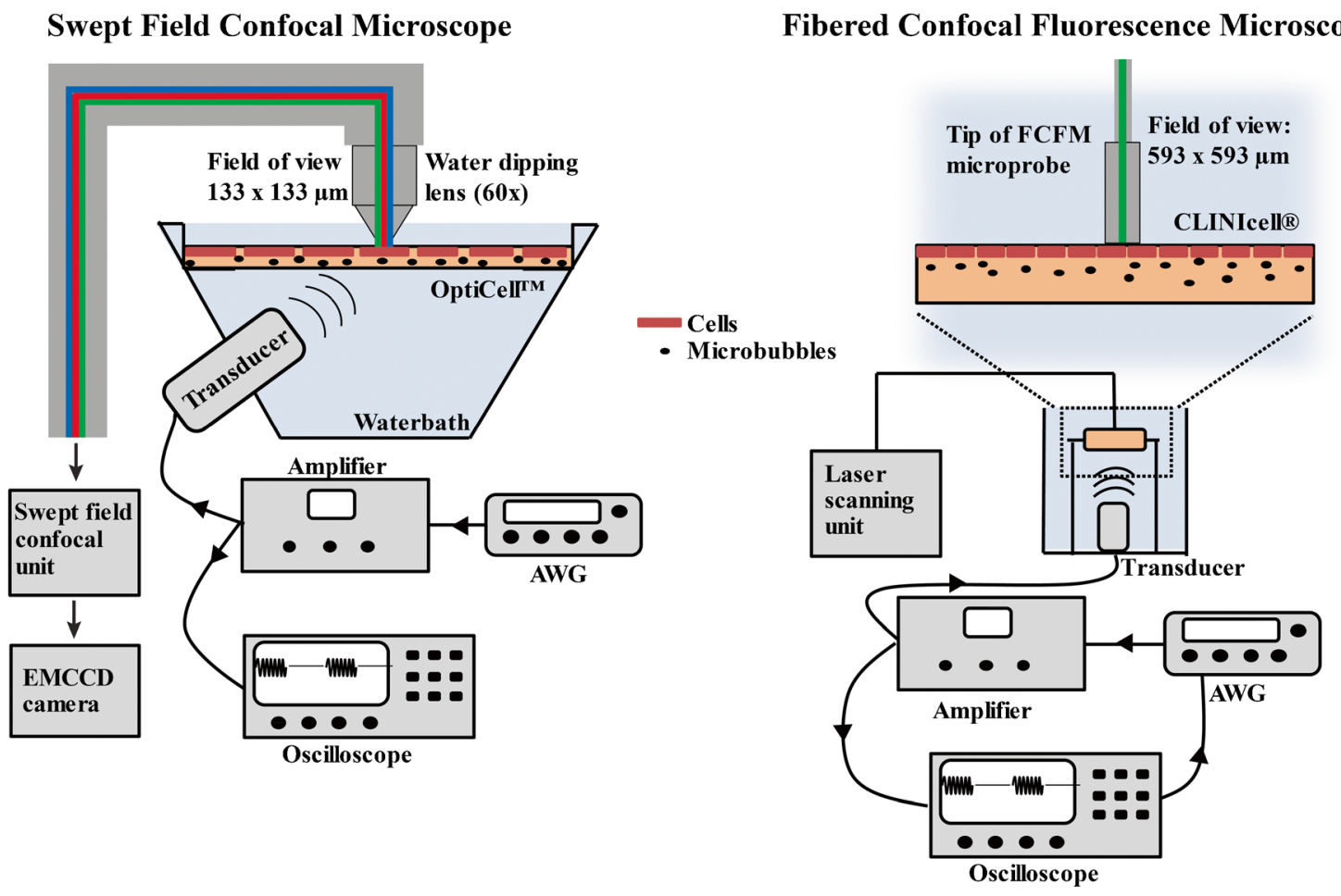

Fig. 1. Schematic representation of the confocal microscopy systems and ultrasound setups used in this study.

monolayer during the experiment, $12 \times 10^{3} \mathrm{C} 6$ cells or $10 \times 10^{3} \mathrm{FaDu}$ cells were seeded per well of a 96-well plate and grown for 2 or 3 days, respectively. Then, cells were washed by PBS and permeabilized by adding $0.5 \%$ Triton X-100 for $1 \mathrm{~min}$. After washing, 1, 2, 5, 10, or $20 \mu \mathrm{M}$ SYTOX Green was added and the well plate was immediately placed in the spectrofluorometer (FP8300, Jasco, Easton, MD, USA). Fluorescent signal enhancement (excitation $504 \mathrm{~nm}$, emission $530 \mathrm{~nm}$ ) was measured every 2 min in FaDu cells and every minute in C6 cells for a total of $180 \mathrm{~min}$. The fluorescence signal of the samples was corrected for the negative control, i.e., cells with PBS only. The relation between the fluorescence rate constant and SYTOX Green concentration was tested by linear regression.

\section{Cell Viability after USMB Exposure}

Viability following USMB exposure was measured via MTS assay in FaDu and C6 cells. In short, $1 \times 10^{6}$ cells were seeded into coated CLINIcells ${ }^{\circledR} 2$ days before the experiments. Then, a mixture of $7 \%(v / v)$ SonoVue ${ }^{\mathrm{TM}}$ microbubbles in $9.5 \mathrm{ml}$ Opti-MEM ${ }^{\circledR}$ was added to the cells, and the complete cell monolayer was exposed by sliding the CLINIcell ${ }^{\circledR}$ over the ultrasound beam using a guidance frame [14]. After allowing cells to recover for $4 \mathrm{~h}$ [12], they were harvested by trypsinization, counted, diluted, and seeded into a 96-well plate. After 3 days, medium was refreshed and $20 \mu \mathrm{l}$ of MTS dye was added
(CellTiter 96® AQueous One Solution Cell Proliferation Assay, Promega, Leiden, Netherlands). Cells were incubated at $37^{\circ} \mathrm{C}$, and absorbance was measured at $492 \mathrm{~nm}$ using a spectrophotometer (Biochrom EZ Read 400, Isogen Life Sciences, Utrecht, Netherlands). The optical density of the samples was corrected for the background signal, i.e., medium and MTS dye without cells.

\section{Data Analysis}

To obtain fluorescence kinetics insensitive to motion, the individual cells in the FCFM data were segmented and tracked over the $30 \mathrm{~min}$ acquisition using MATLAB (MathWorks, USA), to determine the photobleaching rate constant $k_{\mathrm{pb}}$ and the fluorescence rate constant $k_{\mathrm{f}}$, similar to Derieppe et al. [22]. For a detailed description of this method, the reader is referred to the "Electronic Supplementary Material" of this manuscript.

Statistical analysis was performed in GraphPad Prism ( $\mathrm{La}$ Jolla, CA, USA). Means were compared by ANOVA and Tukey's multiple comparison test.

\section{Results}

USMB-induced SYTOX Green uptake was monitored in high spatiotemporal resolution in BLM cells using a swept field confocal microscope. In addition, the fluorescence kinetics were studied in a population of cells as a function of laser duty cycle, dye concentration, and 
$\mathbf{a}$
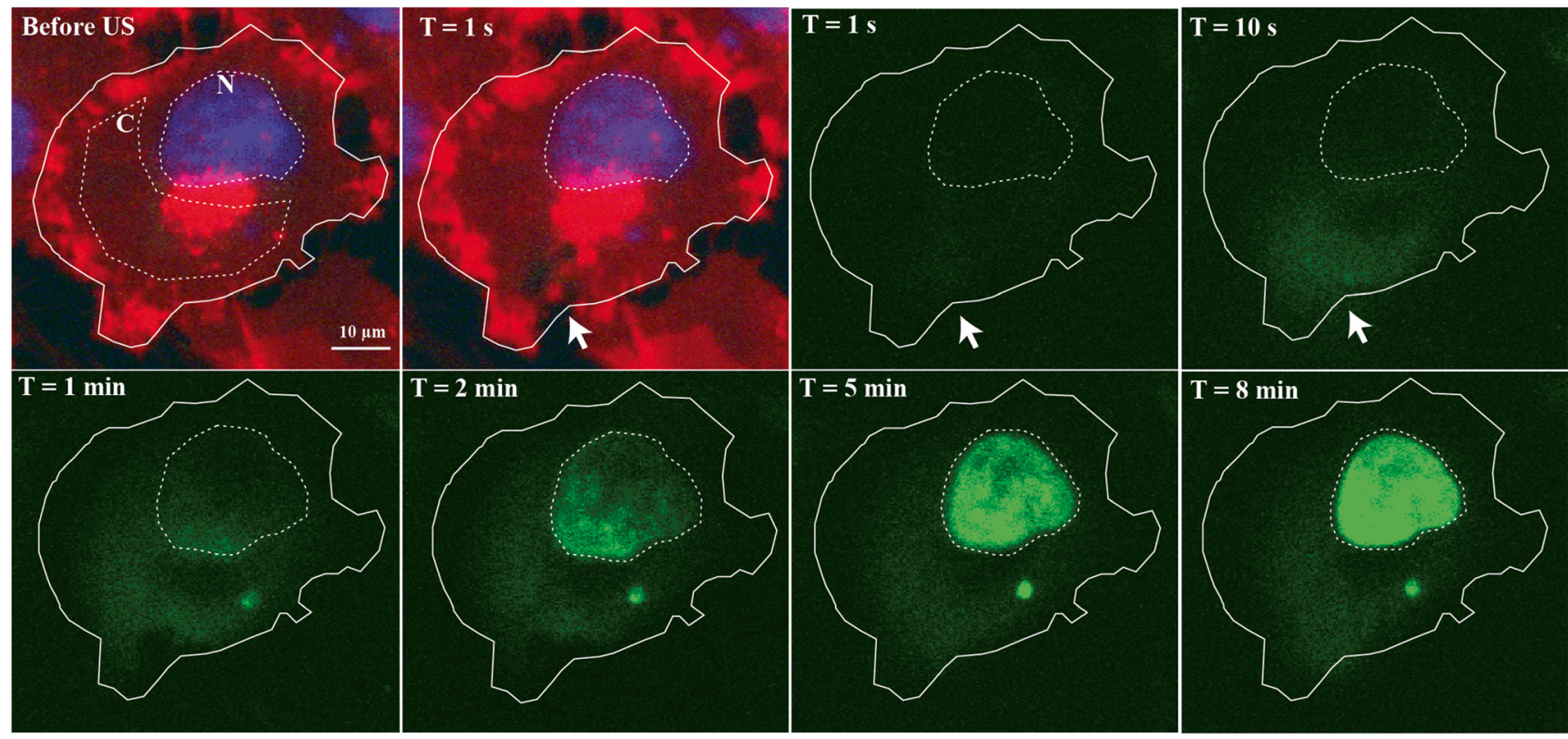

b
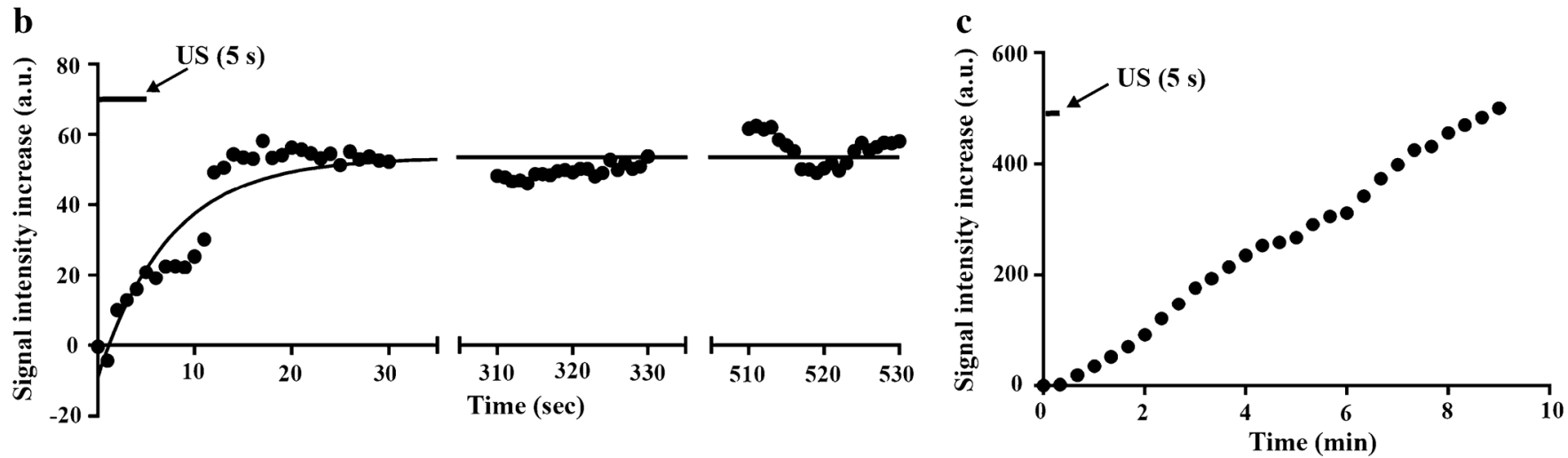

Fig. 2. USMB-induced SYTOX Green uptake. a Time series of confocal images. Cell membrane and nucleus were labeled in red and blue to show the cell morphology before ultrasound was turned on and the pore created by ultrasound (white arrow). SYTOX Green uptake is monitored over the next $8 \mathrm{~min}$ (shown in green). Two regions of interest were drawn in the cell to analyze the SYTOX Green signal in these compartments: $C$ marks the cytosolic region of interest, and $N$ marks the nucleus. Ultrasound was turned on for $5 \mathrm{~s}$ at $T=0$. b Signal intensity time curve of SYTOX Green in the cytosolic region of interest of the BLM cell shown in (a). c Nuclear signal intensity time curve of SYTOX Green in the same cell. For the colored version of the figures, the reader is referred to the online manuscript.

cell line using the fibered confocal fluorescence microscope.

\section{High-Resolution Imaging of USMB-Induced SYTOX Green Uptake in Single Cells}

Swept field confocal microscopy allowed to qualitatively assess the relation between SYTOX Green uptake and fluorescence intensity. Upon ultrasound exposure, a membrane pore is created (Fig. 2a). The intracellular signal around the pore increases in the next $10 \mathrm{~s}$, suggesting that the pore facilitates a local entry of SYTOX Green into the cell. During the subsequent
$50 \mathrm{~s}$, SYTOX Green fluorescence spreads from the pore area further into the cytosol, indicating diffusion through the cytosol. After about $1 \mathrm{~min}$, the fluorescence signal also increases in the nucleus, starting at the side of the membrane pore. Over the next minutes, the fluorescence signal of the nucleus further enhanced and became more homogeneous, most likely representing an increase in SYTOX Green molecules binding to DNA after entering the nucleus. Image analysis demonstrated that mean fluorescence intensity of SYTOX Green in the cytosol increased over a time period of about $15 \mathrm{~s}$ after US was turned on, before it reached a maximum signal plateau (Fig. 2b), while the signal intensity in the nucleus kept increasing during the whole 9-min acquisition (Fig. 2c). 

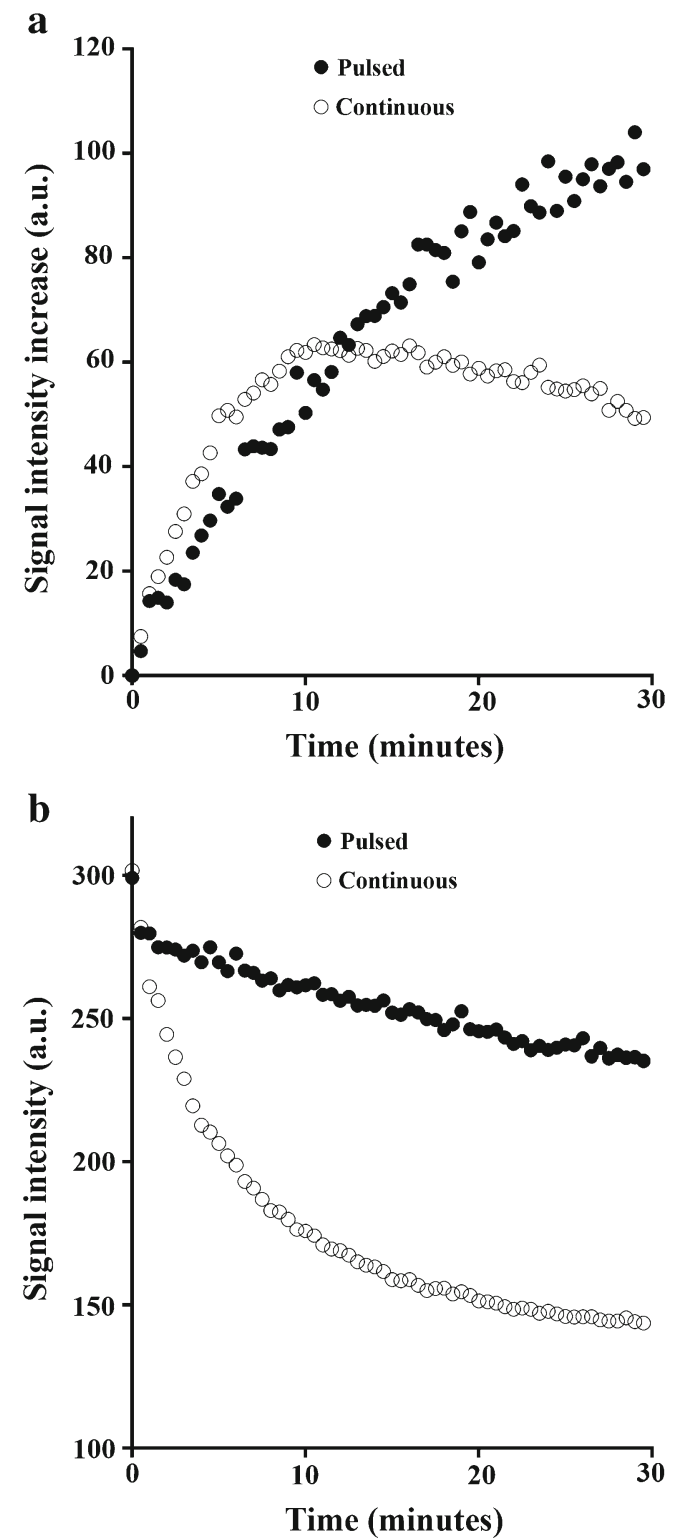

Fig. 3. Fluorescence intensity of representative FaDu cells exposed to $2 \mu \mathrm{M}$ SYTOX Green as a function of time exposed to a pulsed (laser duty cycle of $3.3 \%$ ) or continuous laser. a Fluorescence intensity of $\mathrm{FaDu}$ cells following ultrasound-induced SYTOX Green uptake. b Fluorescence intensity of permeabilized, fixed, and SYTOX Green-stained FaDu cells over time of acquisition.

\section{Influence of Laser-Induced Photobleaching on Fluorescence Kinetics}

The effect of photobleaching on the fluorescence kinetics was investigated by monitoring ultrasound-induced SYTOX Green uptake using the pulsed mode or continuous mode of the FCFM fluorescence laser. While other experimental conditions were identical, continuous laser led to a different signal intensity profile compared to pulsed laser (Fig. 3a) and fluorescence rate constants as fitted with the twocompartment model differed significantly, $1.02( \pm 1.09) \times 10$ ${ }^{-3} \mathrm{~s}^{-1}$ for pulsed laser and $6.53( \pm 3.29) \times 10^{-3} \mathrm{~s}^{-1}$ for continuous laser, respectively.

When trying to correct for photobleaching by using a three-compartment model to fit the fluorescence intensity, an average fluorescent rate constant of $0.34( \pm 0.21) \times 10^{-3} \mathrm{~s}^{-1}$ is obtained, which is substantially different from the pulsed laser results. While the photobleaching correction by the three-compartment model somewhat improved the accuracy of the fluorescence kinetics, it did not completely correct for photobleaching in practice (Table 1).

The laser duty cycle also affected the signal intensity profiles of chemically permeabilized, SYTOX Green positive cells (Fig. 3b). Although both cells had about the same fluorescence intensity at the start of the recording, the cellular SYTOX Green signal intensity decreased much faster when the cell was exposed to continuous laser, compared to pulsed laser, with a photobleaching rate constant $k_{\mathrm{pb}}$ that was 6.4-fold higher for the continuous laser (Table 1). This is equivalent to the 6.4-fold higher fluorescence rate constant $k_{\mathrm{f}}$ of the continuous laser compared to the pulsed laser, suggesting that the different fluorescence intensity time curves of Fig. 3a are primarily the result of photobleaching.

\section{Concentration-Dependent Fluorescence Kinetics}

To investigate the influence of dye concentration on the fluorescence kinetics, $\mathrm{FaDu}$ cells were exposed to USMB in the presence of different concentrations of SYTOX Green, while monitoring uptake in the cell population with FCFM, using the pulsed laser to minimize photobleaching. Linear regression demonstrated that the fluorescence rate constant $k_{\mathrm{f}}$ significantly increased with SYTOX Green concentration $(p<0.05$; Fig. 4a). The fluorescence kinetics showed a

Table 1. Photobleaching rate constants $k_{\mathrm{pb}}$ and fluorescence intensity rate constants $k_{\mathrm{f}}$ of SYTOX Green in FaDu cells

\begin{tabular}{lllll}
\hline & $\begin{array}{l}\text { Pulsed laser } \\
\left(10^{-3} \mathrm{~s}^{-1}\right)\end{array}$ & $\begin{array}{l}\text { Continuous laser } \\
\left(10^{-3} \mathrm{~s}^{-1}\right)\end{array}$ & Ratio & Significance $(p)$ \\
\hline$k_{\mathrm{pb}}$ & $0.41( \pm 0.35)$ & $2.63( \pm 0.31)$ & 6.4 & $<0.0001$ \\
$k_{\mathrm{f}} 2 \mathrm{CM}$ & $1.02( \pm 1.09)$ & $6.53( \pm 3.29)$ & 6.4 & $<0.0001$ \\
$k_{\mathrm{f}} 3 \mathrm{CM}$ & $\mathrm{n} / \mathrm{a}$ & $0.34( \pm 0.21)$ & & \\
\hline
\end{tabular}

Values represent mean \pm standard deviation

$2 C M$ two-compartment model, $3 C M$ three-compartment model, $n / a$ not applicable 

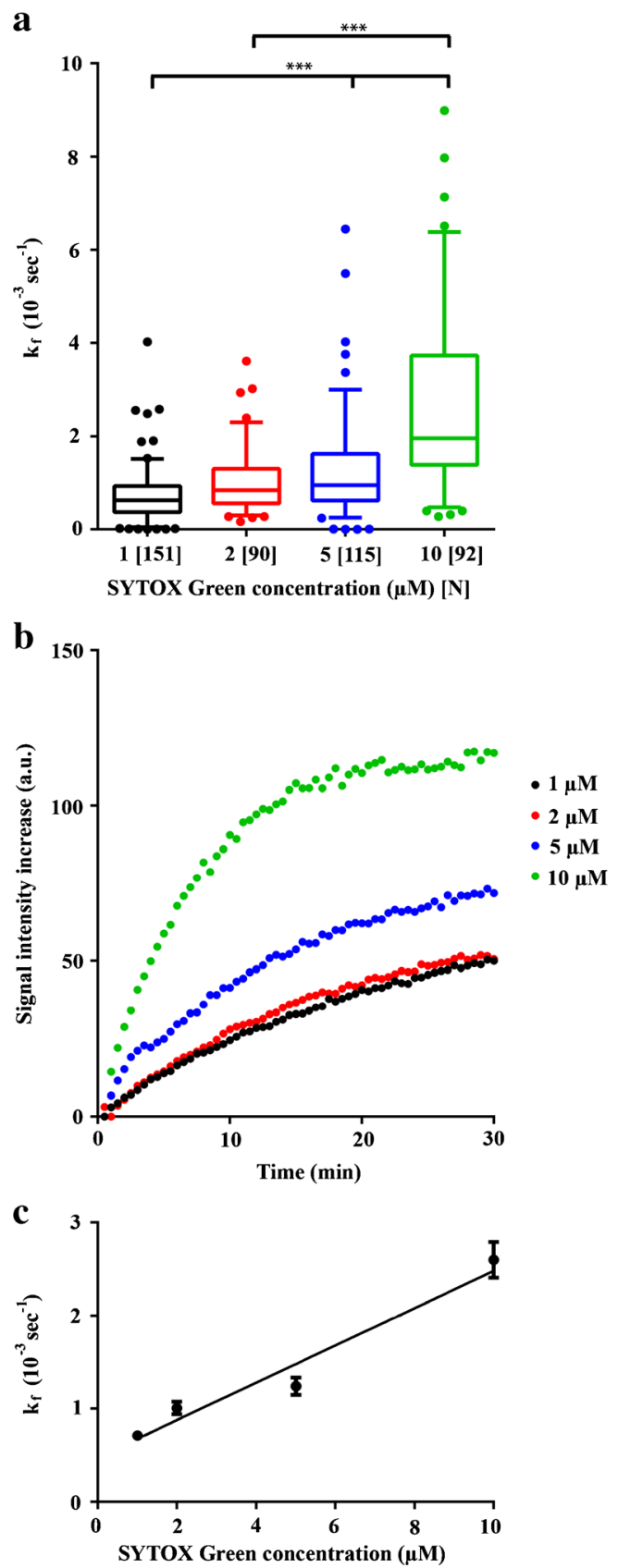

Fig. 4. a Fluorescence rate constants $\left(k_{f}\right)$ as a function of SYTOX Green concentration. Whiskers represent the 5-95 percentile and $N$ the number of cells per group. b Fluorescence signal intensity as a function of time for representative cells that were exposed to ultrasound in the presence of 1, 2, 5 , or $10 \mu \mathrm{M}$ SYTOX Green. c Scatterplot of $k_{\mathrm{f}}$ as a function of SYTOX Green concentration. Symbols represent mean \pm SEM. The three asterisks indicate $p<0.001$.

linear relation with the concentration, described by $k_{\mathrm{f}}=0.2$ $( \pm 0.03) \times 10^{-3} \mathrm{~s}^{-1} \mu \mathrm{M}^{-1}$. In addition, increasing SYTOX Green concentrations resulted in higher signal intensities (Fig. 4b), indicating that for the concentrations evaluated, the available sites for DNA-SYTOX Green binding, which mainly determines the fluorescence signal intensity observed in the nuclei, were not saturated (Fig. 4b).

\section{SYTOX Green Fluorescence Kinetics in Chemically Permeabilized FaDu Cells}

To investigate the relation between SYTOX Green concentration and fluorescence kinetics in a model of membrane permeability where the effect of pore resealing after USMB-induced membrane pores is excluded, FaDu cells were chemically permeabilized and fluorescence intensity was measured over time in a cell population by fluorescence spectrometry. The fluorescence signal enhancement was faster with higher SYTOX Green concentrations (Fig. 5a). In FaDu cells exposed to 1 or $2 \mu \mathrm{M}$ SYTOX Green, fluorescence intensity kept increasing for the duration of the measurement, i.e., $3 \mathrm{~h}$. Similar to the ultrasound experiments, increasing dye concentration resulted in faster signal enhancement and enhanced maximal fluorescence intensity, except for the $20 \mu \mathrm{M}$ SYTOX Green, where we suspect that quenching occurred. Up to $10 \mu \mathrm{M}$, the fluorescence rate constant $k_{\mathrm{f}}$ correlated linearly with SYTOX Green concentration (Fig. 5b), with a concentration dependence of $k_{\mathrm{f}}$ of $0.056( \pm 0.007) \times 10$ ${ }^{-3} \mathrm{~s}^{-1} \mu \mathrm{M}^{-1}$. These fluorescence rate constants are much lower compared to the fluorescence rate constants following USMB-induced permeabilization. This may suggest that the membrane integrity restored faster in USMBexposed cells compared to chemically permeabilized cells. However, caution should be taken when comparing the fluorescence kinetics between USMB and chemical membrane permeabilization, because the method of permeabilization and the analytical technique differed between the experiments.

\section{Cell Line-Dependent Fluorescence Kinetics and Viability}

To investigate if USMB exposure of different cell lines resulted in different fluorescence kinetics, $\mathrm{C} 6-$ and $\mathrm{FaDu}$ cells were exposed to USMB at identical experimental conditions. C6 cells reached maximum fluorescence intensity after $\sim 15 \mathrm{~min}$, while this was $>30$ min for FaDu cells (Fig. 6a). In fact, the median $k_{\mathrm{f}}$ of C6 cells was 2.4-fold higher than the $k_{\mathrm{f}}$ of FaDu cells (Fig. 6b). This long fluorescence signal enhancement was also observed in the swept field confocal microscopy experiments, even though the FCFM used higher acoustic pressures. Moreover, we found no relation between acoustic pressure and fluorescence kinetics in FaDu and $\mathrm{C} 6$ cells, while $\mathrm{C} 6$ cells showed consistently faster kinetics than FaDu cells at each pressure (Suppl. Fig. 1).

Also in chemically permeabilized cells, C6 cells showed faster fluorescence kinetics than FaDu cells (Suppl. Fig. 2). This suggests that the differences between these cell lines cannot be solely attributed to different susceptibility to USMB exposure, e.g., different pore resealing kinetics. 

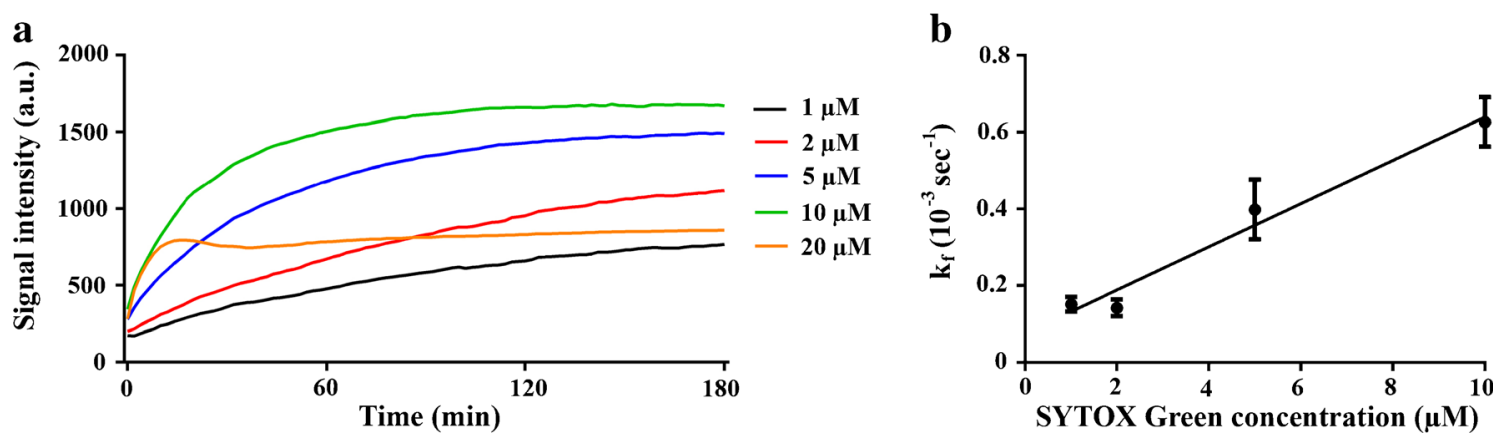

Fig. 5. SYTOX Green uptake and fluorescence signal enhancement of chemically permeabilized FaDu cells. a Average SYTOX Green signal intensity over time after addition of $1,2,5,10$, or $20 \mu \mathrm{M}$ to permeabilized cells $(N=4)$. b Fluorescence rate constants $\left(k_{f}\right)$ of signal intensity after addition of different SYTOX Green concentrations to permeabilized cells as a function of SYTOX Green concentration. Symbols represent mean \pm SEM $(N=4)$.

The long-term viability of FaDu and C6 cells was verified via MTS assay, to rule out that the measured SYTOX Green uptake which was the result of irreversibly sonoporated cells. Fig. 6c demonstrates that USMB exposure of FaDu cells resulted in a viability that was slightly, though significantly, lower than control cells $(p<0.05)$, while the viability of C6 cells was not hampered by USMB $(p>0.05)$. This demonstrates that USMB-induced SYTOX Green uptake measured by FCFM was primarily the result of reversible membrane permeability.

\section{Discussion}

The goal of this study was to characterize the fluorescence signal enhancement after intracellular uptake of SYTOX Green, with regard to the effects of laser duty cycle, dye concentration, and cell line. First, we observed that USMB-induced pore formation resulted in intracellular signal enhancement of SYTOX Green, spreading throughout the cell and ultimately accumulating in the nucleus. By systematically varying experimental parameters, we have demonstrated that the fluorescence kinetics were substantially influenced by laser duty cycle, dye concentration, and cell line.

Membrane impermeant intercalating model drugs like SYTOX Green [12], PI [23], or TOTO-3 [24] are widely used to study USMB-induced intracellular uptake. Due to their large fluorescence enhancement upon binding to nucleic acids, these model drugs display high intracellular and very low extracellular signal intensity, making them useful agents to study cellular internalization.

However, problems emerge when studying the kinetics of intracellular model-drug uptake, as the fluorescence enhancement reflects not only cellular uptake but also intracellular diffusion, nuclear accumulation, and DNA binding. We observed that USMB-induced pore formation resulted in a local entry of SYTOX Green into the cell, similar to previous work with SYTOX Green [25] or PI [10]. Moreover, others have linked the location of a specific microbubble with the location of cellular entry of PI $[6,17$, 18]. In our studies, intracellular SYTOX Green signal was seen to spread throughout the cell and ultimately accumulated in the nucleus, which involves intracellular diffusion and DNA binding. The nuclear fluorescence signal increased for more than $9 \mathrm{~min}$, while the cytosolic signal intensity reached a maximum after $15 \mathrm{~s}$. Recently, Helfield et al., made a similar observation, that the cytosol reached maximum fluorescence intensity much faster than the nucleus after USMB exposure in the presence of PI [26]. The fluorescence kinetics of the cytosol may thus hold a more direct association with the membrane permeability, e.g., pore resealing, whereas the nuclear fluorescence kinetics is influenced by additional processes, such as intracellular diffusion and nuclear accumulation. However, the fast plateau of fluorescence intensity in the cytosol may also be influenced by saturation of cytosolic nucleic acids.

Furthermore, signal intensity was much higher in the nucleus compared to the cytosol, most likely as a result of the higher nucleic acid concentration in the nuclear compartment, which can thus bind larger quantities of SYTOX Green. When cellular signal intensity enhancement following USMB treatment is monitored in low spatial resolution, such as with FCFM, the signal from the nucleus is dominant. Therefore, it is not surprising that fluorescence kinetics of the cellular signal on FCFM and the nuclear signal on the swept field confocal microscope were highly similar. The swept field confocal microscope allows us to monitor SYTOX Green signal enhancement with subcellular precision, but there were only a few cells that showed a clear diffusion pattern as shown in Fig. 2. FCFM on the other hand allows studying fluorescence kinetics on a larger scale, typically a population of hundreds of cells at a time.

Fluorescence kinetics were substantially influenced by the laser duty cycle. Although SYTOX Green shows relatively low photobleaching [27]; the continuous laser induced a sixfold enhancement of the photobleaching rate, which resulted in a considerable overestimation of the fluorescence kinetics, that could not be corrected by using a three-compartment model fit of the signal. Therefore, we preferred to minimize the effect of photobleaching by 

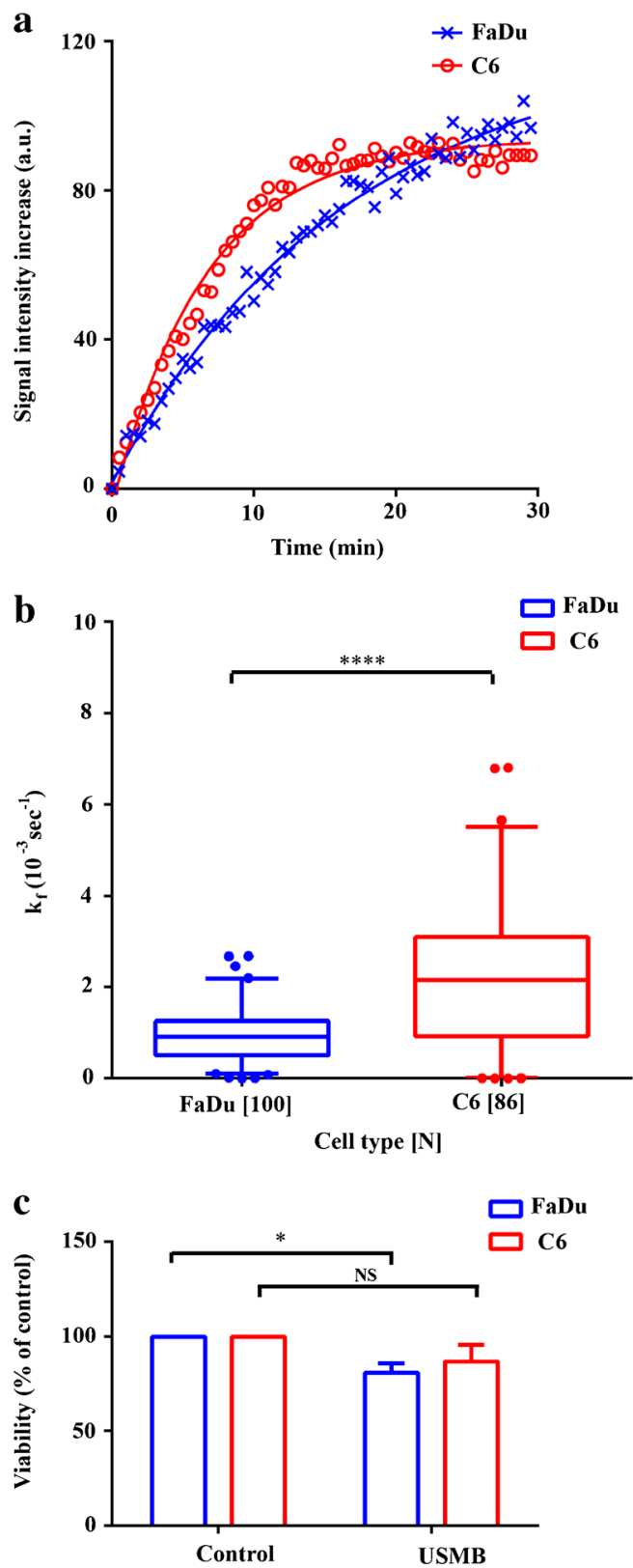

Fig. 6. a Representative SYTOX Green signal intensity curves over time of $\mathrm{FaDu}$ and $\mathrm{C} 6$ cells following exposure to $850 \mathrm{kPa}$ ultrasound. b Fluorescence rate constants $\left(k_{\mathrm{f}}\right)$ versus acoustic pressure for FaDu and $\mathrm{C} 6$ cells. Cells were exposed to ultrasound in the presence of $2 \mu \mathrm{M}$ SYTOX Green. Whiskers represent the 5-95\% percentile. c Viability of FaDu and C6 cell USMB treatment, measured via MTS assay. Bars represent mean + standard deviation $(N=3)$. NS not significant. One asterisk indicates $p<0.05$, three asterisks indicate $p<0.001$, and four asterisks indicate $p<0.0001$.

recording the fluorescence signal enhancement with a pulsed laser only. Obviously, the frame rate of pulsed recordings must be high enough to sample the fluorescence signal enhancement. Alternatively, anti-fading agents could be employed to reduce photobleaching [28], but the effect of this approach was not tested in this study.

We found similar fluorescence kinetics between SYTOX Green and PI (Suppl. Fig. 3), while others have reported faster fluorescence kinetics for PI [17, 18]. This is most likely due to the higher PI concentration used in these studies. For example, Fan et al. used an extracellular PI concentration of $100 \mu \mathrm{M}$ and demonstrated that cellular PI signal intensity increased for 1 to 5 min post-sonication before reaching maximum signal intensity. This is in line with the concentration-dependent fluorescence kinetics found in our study, i.e., $k_{\mathrm{f}}=0.2( \pm 0.03) \times 10^{-3} \mathrm{~s}^{-1} \mu \mathrm{M}^{-1}$, where $100 \mu \mathrm{M}$ dye would result in a fluorescent rate constant of $20 \times 10^{-3} \mathrm{~s}^{-1}$. This corresponds to reaching $67 \%$ of the maximal fluorescence intensity in $50 \mathrm{~s}$, a similar kinetic pattern as observed by Fan et al. Nevertheless, they attributed the time window of fluorescence signal enhancement to the time needed for pore resealing, while assuming instantaneous binding of PI to cellular RNA or DNA [17]. More recently, van Rooij et al. also showed fast uptake kinetics of PI following USMB ( $<2 \mathrm{~min})$, while using a much lower concentration of PI $(37 \mu \mathrm{M})$ [19]. Similar to Fan et al., they related the time of PI signal enhancement after sonication to pore resealing and attributed signal enhancement beyond $120 \mathrm{~s}$ to cell death, which, based on our findings, may also occur in viable cells.

Although the time window of PI signal enhancement following ultrasound exposure has been associated with pore resealing, we demonstrated that this is primarily dependent on experimental parameters, such as laser duty cycle and dye concentration. Fluorescent signal enhancement is much slower at lower dye concentrations, which has been interpreted in some cases as membrane pores being open for much longer. Still, the uptake kinetics of model drugs can be studied using fluorescence intensity enhancement when using identical experimental conditions throughout experiments, but researchers should be aware of the influence of the experimental parameters on the apparent uptake kinetics.

Direct microscopic observations of ultrasound-induced membrane pores have shown that resealing occurs within seconds up to a minute [29]. Therefore, we speculate that during the time that pores are open, SYTOX Green molecules enter the cell, while the pore reseals within a minute. The quantity of intracellular SYTOX Green depends primarily on the extracellular concentration. Subsequently, SYTOX Green diffuses throughout the cell and accumulates in the nucleus by binding to DNA. The rate of diffusion is influenced by the concentration, in accordance with Fick's law. Similarly, the statistical nature of DNA intercalator binding makes that this rate is influenced by the concentration of the intercalator as well as the number of available binding sites. Furthermore, additional SYTOX Green may enter the cell after the pores have resealed, e.g., by endocytosis $[11,30]$. Together, these events result in the signal intensity enhancement, which can last much longer than the time during which the pores remain open. 
Alternative methods may also be explored to investigate the uptake kinetics of (model) drugs following USMB therapy. Non-intercalating model drugs like Green Fluorescent Protein (GFP) or FITC-dextrans can be used to exclude the effect of nucleic acid binding. However, as these dyes display high background signal, they are difficult to use for monitoring USMB-induced intracellular influx in real time. In addition, such an approach will face similar limitations as this study, including the semi-quantitative nature of the optically measured kinetic data. Alternatively, intracellular (model) drug concentrations can be quantitatively measured and validated at discrete timepoints, i.e., not in real-time, post-sonication by, e.g., HPLC, but for fluorescent intercalating compounds, the dependence of the HPLC detection on fluorescence is confounding. Furthermore, the transient membrane invaginations resulting from USMB can be studied by transmission electron microscopy or scanning electron microscopy [13], which provide useful insight in the structural changes of the membrane and the timescale of membrane recovery. However, these techniques do not allow monitoring (model drug) transport across the membrane. Lastly, instead of measuring USMB-induced model drug influx, the efflux of an intracellular dye, such as GFP, can be measured by fluorescence microscopy, which can be associated with pore resealing and exocytosis [31].

\section{Conclusion}

This study demonstrated that the kinetics of fluorescence enhancement following ultrasound-induced fluorescent model drug uptake were not only associated with membrane pore resealing but also with intracellular diffusion and accumulation in the nucleus. In addition, we showed that the fluorescence kinetics were substantially influenced by experimental parameters, such as the laser duty cycle, dye concentration and cell line. Therefore, as SYTOX Green fluorescence enhancement is governed by several mechanisms and largely influenced by experimental parameters, its fluorescence kinetics should be carefully interpreted before relating it to biological processes, such as pore resealing. Specifically, the results from experiments and studies using different dye concentrations or fluorescence imaging systems should not be compared directly.

Acknowledgements. This work was financially supported by the Advanced European Research Council Grant "Sound Pharma"-268906 (CM).

\section{Compliance with Ethical Standards}

\section{Conflict of Interest}

The authors declare that they have no conflict of interest.

Open Access This article is distributed under the terms of the Creative Commons Attribution 4.0 International License (http:// creativecommons.org/licenses/by/4.0/), which permits unrestricted use, distribution, and reproduction in any medium, provided you give appropriate credit to the original author(s) and the source, provide a link to the Creative Commons license, and indicate if changes were made.

\section{References}

1. Frenkel V (2008) Ultrasound mediated delivery of drugs and genes to solid tumors. Adv Drug Deliv Rev 60:1193-1208

2. Allen TM, Cullis PR (2004) Drug delivery systems: entering the mainstream. Science 303:1818-1822

3. Han HK, Amidon GL (2000) Targeted prodrug design to optimize drug delivery. AAPS PharmSci 2:48-58

4. Lentacker I, De Cock I, Deckers R et al (2014) Understanding ultrasound induced sonoporation: definitions and underlying mechanisms. Adv Drug Deliv 72:49-64

5. Escoffre JM, Piron J, Novell A, Bouakaz A (2011) Doxorubicin delivery into tumor cells with ultrasound and microbubbles. Mol Pharm 8:799-806

6. van Wamel A, Kooiman K, Harteveld M et al (2006) Vibrating microbubbles poking individual cells: drug transfer into cells via sonoporation. J Control Release 112:149-155

7. Tachibana K, Uchida T, Ogawa K et al (1999) Induction of cellmembrane porosity by ultrasound. Lancet 353:1409

8. Deng CX, Sieling F, Pan H, Cui J (2004) Ultrasound-induced cell membrane porosity. Ultrasound Med Biol 30:519-526

9. Meijering BDM, Juffermans LJM, van Wamel A et al (2009) Ultrasound and microbubble-targeted delivery of macromolecules is regulated by induction of endocytosis and pore formation. Circ Res 104:679-687

10. De Cock I, Zagato E, Braeckmans K et al (2015) Ultrasound and microbubble mediated drug delivery: acoustic pressure as determinant for uptake via membrane pores or endocytosis. J Control Release 197:20-28

11. Zeghimi A, Escoffre JM, Bouakaz A (2015) Role of endocytosis in sonoporation-mediated membrane permeabilization and uptake of small molecules: a electron microscopy study. Phys Biol 12:66007

12. Lammertink BHA, Deckers R, Storm G et al (2015) Duration of ultrasound-mediated enhanced plasma membrane permeability. Int $\mathrm{J}$ Pharm 482:92-98

13. Brayman AA, Coppage ML, Vaidya S, Miller MW (1999) Transient poration and cell surface receptor removal from human lymphocytes in vitro by $1 \mathrm{MHz}$ ultrasound. Ultrasound Med Biol 25:999-1008

14. Lammertink BHA, Bos C, van der Wurff-Jacobs KM et al (2016) Increase of intracellular cisplatin levels and radiosensitization by ultrasound in combination with microbubbles. J Control Release 238:157-165

15. Zhong W, Chen X, Jiang P et al (2013) Induction of endoplasmic reticulum stress by sonoporation: linkage to mitochondria-mediated apoptosis initiation. Ultrasound Med Biol 39:2382-2392

16. Derieppe M, Yudina A, Lepetit-Coiffé M et al (2013) Real-time assessment of ultrasound-mediated drug delivery using fibered confocal fluorescence microscopy. Mol Imaging Biol 15:3-11

17. Fan Z, Liu H, Mayer M, Deng CX (2012) Spatiotemporally controlled single cell sonoporation. Proc Natl Acad Sci 109:16486-16491

18. Qin P, Xu L, Han T et al (2016) Effect of non-acoustic parameters on heterogeneous sonoporation mediated by single-pulse ultrasound and microbubbles. Ultrason Sonochem 31:107-115

19. van Rooij T, Skachkov I, Beekers I et al (2016) Viability of endothelial cells after ultrasound-mediated sonoporation: influence of targeting, oscillation, and displacement of microbubbles. J Control Release 238:197-211

20. Quax PH, van Muijen GN, Weening-Verhoeff EJ et al (1991) Metastatic behavior of human melanoma cell lines in nude mice correlates with urokinase-type plasminogen activator, its type-1 inhibitor, and urokinasemediated matrix degradation. J Cell Biol 115:191-199

21. de Chaumont F, Dallongeville S, Chenouard N et al (2012) Icy: an open bioimage informatics platform for extended reproducible research. Nat Methods 9:690-696

22. Derieppe M, de Senneville BD, Kuijf H et al (2014) Tracking of cell nuclei for assessment of in vitro uptake kinetics in ultrasoundmediated drug delivery using fibered confocal fluorescence microscopy. Mol Imaging Biol 16:642-651

23. Fan Z, Kumon RE, Park J, Deng CX (2010) Intracellular delivery and calcium transients generated in sonoporation facilitated by microbubbles. J Control Release 142:31-39 
24. Deckers R, Yudina A, Cardoit LC, Moonen CTW (2011) A fluorescent chromophore TOTO-3 as a 'smart probe' for the assessment of ultrasound-mediated local drug delivery in vivo. Contrast Media Mol Imaging 6:267-274

25. Leow RS, Wan JMF, Yu ACH (2015) Membrane blebbing as a recovery manoeuvre in site-specific sonoporation mediated by targeted microbubbles. J R Soc Interface 12:20150029

26. Helfield B, Chen X, Watkins SC, Villanueva FS (2016) Biophysical insight into mechanisms of sonoporation. Proc Natl Acad Sci U S A 113:9983-9988

27. Thakur S, Cattoni DI, Nöllmann M (2015) The fluorescence properties and binding mechanism of SYTOX green, a bright, low photo-damage DNA intercalating agent. Eur Biophys J 44:337-348
28. Longin A, Souchier C, Ffrench M, Bryon PA (1993) Comparison of anti-fading agents used in fluorescence microscopy: image analysis and laser confocal microscopy study. J Histochem Cytochem $41: 1833-1840$

29. Hu Y, Wan JMF, Yu ACH (2013) Membrane perforation and recovery dynamics in microbubble-mediated sonoporation. Ultrasound Med Biol 39:2393-2405

30. Derieppe M, Rojek K, Escoffre JM et al (2015) Recruitment of endocytosis in sonopermeabilization-mediated drug delivery: a realtime study. Phys Biol 12:46010

31. Kaddur K, Lebegue L, Tranquart F et al (2010) Transient transmembrane release of green fluorescent proteins with sonoporation. IEEE Trans Ultrason Ferroelectr Freq Control 57:1558-1567 